\title{
Allergic Rhinitis and Its Impact on Sleep
}

\author{
J. Rimmer ${ }^{1}$ and J. Hellgren ${ }^{2}$ \\ ${ }^{1}$ Woolcock Institute and University of Sydney, Department of ENT, Sydney \\ ${ }^{2}$ Head \& Neck Surgery, Sahlgrenska Academy, Göteborg \\ ${ }^{1}$ Australia \\ ${ }^{2}$ Sweden
}

\section{Introduction}

Allergic rhinitis affects a fifth of the population in industrialised countries and sleep disturbance has emerged as one of the major impacts of allergic rhinitis on patients. Sleep disturbances and its consequences on daytime sleepiness and fatigue affects the health related quality of life negatively and contributes significantly to patients'suffering and major health economic costs. Experimental studies have shown that sleep disturbances are induced when the nose is blocked with adhesive tape or petroleum jelly during sleep in healthy individuals. Nasal obstruction is the most commonly reported symptom by patients with allergic rhinitis and it causes an increased number of microarousals (short awakenings). Nasal obstruction is believed to be the most important mechanism behind poor sleep and daytime sleepiness in allergic rhinitis. Relieving nasal obstruction with nasal steroids significantly improves subjective sleep compared to placebo. Allergic rhinitis is present in a majority of patients with asthma. Identifying and treating allergic rhinitis improves sleep and the health related quality of life which reduces patient suffering and potentially saves significant health economic health costs. The present chapter reviews the current literature of allergic rhinitis and its impact on sleep and the health related quality of life.

\subsection{Abbreviations}

AR: allergic rhinitis

PAR: perennial allergic rhinitis

SAR: seasonal allergic rhinitis

SDB: sleep disordered breathing

RDS: rhinitis disturbed sleep

\subsection{Prevalence}

The prevalence of allergic rhinitis has steadily increased in developed countries since the industrial revolution and now affects $20-40 \%$ of the population $(1,2)$. Studies suggest that allergic rhinitis and conjunctivitis are rare in infants but are estimated to affect around one in six children aged 6-7 years, one in ten children aged $13-14$ years, $18 \%$ of those aged $15-34$ years and $10 \%$ of older adults aged $35-54$ years. AR is more common than asthma and chronic rhinosinusitis. Symptoms generally persist for at least ten years, often longer (3). Sleep disordered breathing has been recorded in $68 \%$ of PAR patients and $48 \%$ with SAR (4). 


\subsection{Costs}

More than $\$ 6$ billion was spent on prescription medications for AR in 2000 (5) in the USA. A specifically commissioned report in Australia estimated the total costs costs of Allergic disease (not asthma) including prescription medication to be $\$ 349$ million in Australia in 2007 (3).

\subsection{Symptoms}

Symptoms of AR include nasal blockage or congestion occurring in up to $85 \%$, and this tends to be the dominant symptom in children (5). It is generally most troublesome in the early morning on waking which may relate to the circadian rhythm for cortisol although the peak in symptoms is delayed compared to that of nocturnal asthma (6am versus 4am) (6). Nasal obstruction is the symptom that relates most to impaired quality of life (7). In addition there is a direct relationship between sleep impairment and symptom severity $(5,8)$. Nasal obstruction has been shown to be an independent risk factor for OSAS (obstructive sleep apnoea syndrome) (9). Other symptoms include sneezing, pruritis, anterior rhinorrhoea, post nasal drip and these may also contribute to SDB.

Numerous studies have shown that AR results in impaired QOL and affects numerous activities eg work productivity and attendance, physical activity, exam performance. How much of this is due to the disease itself or the accompanying SDB or both is unclear.

Interestingly many patients report dissatisfaction with the effectiveness of therapy (10).

\subsection{Children and AR}

SDB is well documented to occur in children and adolescents with AR $(5,11)$ The most common symptom is nasal obstruction. In children there are typical appearances associated with AR including mouth breathing, allergic shiners, allergic crease, allergic facies and these may be reversed by treatment of the rhinitis. Rhinitis has frequently been considered to be a benign condition in children and one that does not need treating. However more recent data indicates that in addition to symptoms there may be deleterious effects of the condition on their performance. Specifically in children studies have shown reduced examination performance in the spring season and reduced participation in skill based, social and informal activities $(12,13)$. Habitual snoring is increased in children with AR (14)

\section{Sleep problems in AR}

Sleep disordered breathing is reported by subjects with allergic rhinitis versus controls including difficulty getting to sleep ( $24 \%$ versus $8 \%$ ), waking during the night ( $31 \%$ versus $13 \%)$ and poor sleep $(26 \%$ versus $11 \%)(5,15)$ Leger et al additionally noted early wakening in $29 \%$ of AR, feeling of lack of sleep in $63 \%$, snoring $40 \%$, insomnia $36 \%$, OSAS $4 \%$ and also that there was a greater use of sedatives in the AR group (16). Poor sleep is associated with negative effects on mood, cognition and motor performance. A large epidemiological study of over 4900 subjects showed that AR subjects who reported nocturnal nasal congestion $\geq 5$ nights/month were more likely to be habitual snorers, experience excess daytime sleepiness or nonrestorative sleep (17). SDB is felt to relate to nasal obstruction but can also be caused by other symptoms eg rhinorrhea, pruritis and sneezing occurring during the night and also by the effects of inflammatory mediators. In addition commonly co-existing diseases such as asthma in which nocturnal asthma can be a significant symptom can disturb sleep. Nocturnal asthma is attributed to a circadian variation in airway physiology as well as the 
effects of cytokine changes on sleep (see below).Eczema may also contribute to SDB with symptoms of pruritis often worse at night (18).

Sleep impairment in AR has been demonstrated by means of questionnaires, actigraphy $(19,20)$ and polysomnography $(17,21)$

The presence of abnormal sleep in association with AR categorises the disease as moderate to severe according to the ARIA classification of AR (Allergic Rhinitis and its Impact on Asthma) guidelines (Fig 1) (22)

\begin{tabular}{|c|c|c|}
\hline Intermittent symptoms & & Persistent symptoms \\
\hline$\bullet<4$ days per week & & $\bullet>4$ days per week \\
\hline$\bullet$ Or $<4$ weeks & & $\bullet$ And $>4$ weeks \\
\hline Mild & & Moderate - severe $(\geq \mathbf{1}$ item) \\
\hline$\bullet$ Normal sleep & & $\begin{array}{l}\text { Impairment of daily } \\
\text { activities, sport, leisure }\end{array}$ \\
\hline$\bullet$ Normal daily activities & & $\begin{array}{l}\text { Problems caused at school } \\
\text { or work }\end{array}$ \\
\hline$\bullet$ Normal work and school & & \begin{tabular}{l} 
Troublesome symptoms \\
\hline
\end{tabular} \\
\hline
\end{tabular}

Fig. 1. ARIA classification of AR

The average sleep duration of healthy adults is about 7 hours but surveys show that a significant \% of the population (43\%) achieve less than this (23) and that these subjects felt tired, performed inefficiently and reported feeling drowsy while driving. It has been estimated that $20 \%$ of all traffic accidents in industrialised societies are related to sleep disorders.

Sleep disorders refers to a range of disorders including insomnia, OSAS, narcolepsy and idiopathic hypersomnolance, periodic limb disorders of sleep and restless leg syndrome. All of these primary sleep disorders are associated with excess daytime fatigue. SBD in AR includes insomnia and OSAS although recently a separate classification of RDS (rhinitis disturbed sleep) has been postulated (24).

Insomnia is a subjective perception of the amount or quality of sleep. It can comprise delayed initiation of sleep, difficulty with sleep maintenance and early awakening. It is associated with impaired QOL as demonstrated by reduced daytime alertness, lethargy, reduced cognitive functioning and altered emotional states. The 2008 NSF Sleep in America Poll found that $11 \%$ of the population described insomnia ( $26 \%$ difficulty falling asleep, $42 \%$ waking during the night, $29 \%$ woken early and unable to return to sleep)(25). Subjects with insomnia have a higher rate of traffic accidents: $5 \%$ versus $2 \%$ of normal sleepers.

OSAS in the general population is estimated at $2-4 \%$ and results in snoring, severe daytime sleepiness and increased risk of traffic accidents. The risk of RTA for OSAS is 12 times greater than controls and also higher than insomniacs. There is a direct relationship between the apnoea-hypopnoea index and crash risk.

However despite the documented impaired cognition and presence of SDB in AR no studies of driving crash risk have been performed. 
RDS is a term that has been suggested to separate the effects of nasal obstruction and pharyngeal obstruction on SDB (24). It has frequently been observed by ourselves and others that the effect of INCS improves nasal related symptoms more consistently and effectively than sleep related objective measures such as the apnoea-hyponoea index and actigraphy measurements (unpublished data,20,24). Therefore effects of INCS on nasal inflammation may result in improvements in subjective sleep indices without improvements in objective sleep measures, supporting the concept of two co-existing conditions. One study undertook polysomnography pre and post pollen season in 25 subjects SAR and 25 normals Subjects with SAR showed a significant increase in symptoms (overall symptoms score 1.08 \pm 2.7 at baseline compared with $21.3 \pm 13.1$ in season) and also subjective increases in daytime sleepiness which only occurred in the moderate and severe SAR subjects. Objective sleep parameters showed an increase in objective sleep abnormalities, but the differences occurred within the normal range and were not felt to be of clinical relevance (26). This study tends to support the fact that RDS may be more significant in AR than other causes of SDB such as OSAS and insomnia.

\section{Mechanisms of sleep impairment in AR}

\subsection{Nasal anatomy and physiology}

Introduction: The nose is an integrated part of the combined upper and lower airways both in function and inflammatory airways disease such as allergic rhinitis and asthma. There has been an increased focus on the significance of nasal breathing and it's effect on health related quality of life and sleep during the last decade. Though easily accessible for examination, still little is known about how the regulation of nasal function is disturbed during inflammatory nasal disease.

George Catlin, a famous American painter, who spent years living with the native north American Indians, found that the Indians did not sleep with an open mouth and he described the relationship between a patent nose and good sleep in his book "Shut your mouth and save your life" in 1832. Respiratory function during sleep is a dynamic process involving changes in respiratory drive, airway patency and in airway muscular tone during REM and non REM sleep which makes the relationship between nasal function and poor sleep complex and difficult to assess. Most studies addressing nasal patency in inflammatory airways disease have been performed in awake subjects sitting in the upright position. In reality the nasal mucosa is highly reactive and responds almost instantly to changes in temperature, humidity and a change in body position. In the evaluation of patients with sleep disturbances and allergic rhinitis several aspects of nasal anatomy and physiology thus have to be taken into account.

External nose: The external nose protrudes from the bone aperture and is mainly composed of cartilage, muscle and subcutaneous fat apart from the two nasal bones at the top. The inside of the nasal openings and the anterior part of the nose is covered with skin and thus unaffected by nasal inflammation. The isthmus, is the narrow opening on the inside of the nose corresponding to the insertion of the nasal wings (alae) and the naso labial crest on the outside. More than half of the airway resistance is under normal conditions located here. During inspiration, the nasal openings tends to collapse as the passage of air through the narrow isthmus area put a suction force on the airway walls, the so called Bernouille effect. Patients with narrow nasal openings and weak alae are more prone to alar collapse especially when it combines with other abnormalities such as a septal deviation, turbinate hypertrophy or 
inflammatory nasal disease. An activation of muscles in the anterior part of the nose help to counteract this tendency to collapse. When air is passed through the narrow isthmus with a high flow rate into the much larger nasal cavity where the flow rate is lower, the air flow becomes turbulent and disperses in a similar fashion to when water is expelled through a garden hose spray nozzle. Through this design the contact area between the air and the walls of the nasal cavity increases and enables the nose to effectively condition the inhaled air.

The nasal cavities: The contact surface between the inhaled air and the mucous membrane is further enhanced by the folded structures on the lateral wall of the nasal cavity called the turbinates. There are three sets of turbinates in each nasal cavity. Most of the nasal cavity is encased in the skull bone and from an anterior view it is pyramid shaped. The inferior turbinates protrude into the airway at the base of the pyramid and an increase in the swelling of the mucosa covering the inferior turbinate can thus effectively obstruct the main nasal air flow like a cork seals a bottle neck.

The nasal mucosa: The nasal respiratory mucosa replaces the skin starting at the head of the inferior turbinate and goes all the way to the nasopharynx. The nasal mucosa has major similarities with the respiratory mucosa of the bronchi and is today considered to be a linked functional organ with the lower respiratory tract which becomes evident in asthma and allergic inflammatory disease. The main difference is the presence of smooth muscle in the bronchi and the presence of sinusoids in the nose. Smooth muscle contraction can cause airway narrowing in the lungs which can be relieved with $\beta$ agonists, but these drugs have no effect in the nose. Nasal patency is mainly regulated by variation of blood content in the erectile capacitance vessels called the sinusoids located in the sub mucosa. The regulation of nasal patency in the sinusoids is mediated through a neurovascular mechanism with different triggers such as air temperature, posture and physical exercise (27). Blood can be shifted in and out of the sinusoids through a rich capillary network equipped with artery-venous shunts. Nasal patency is maintained by a continuous sympathetic tone that can be up or down regulated. Increased sympathic activity during physical exercise or addition of adrenergic agonists increase nasal patency while a decrease in sympathetic activity decrease nasal patency as in Horner's syndrome. In a third of the population "the nasal cycle" causes an alternating congestion and decongestion of the two nasal cavities going from one side to the other, a few hours apart. Nasal patency can also be changed by the application of pressure to the body surface. Unilateral pressure to the axillary region in the sitting position results in nasal congestion on the ipsilateral side and decongestion on the contralateral side, mediated via pressure sensitive receptors in the skin (28). A change in body position from sitting to supine also changes nasal patency, which may be important in regulating nasal patency during sleep as will be discussed in detail further down.

Nasal function: Breathing through the nose conditions the inhaled air which increases in humidity and temperature. Particles are cleared and the immune system is activated against inhaled bacteria and viruses to protect the lungs. Nitric oxide from the nasal and sinus mucosa is added to the inhaled air which promotes the gas exchange in the lungs by enhancing the ventilator-perfusion ratio. During nasal exhalation humidity and energy is effectively recovered from the air compared to the oral route (29). Nasal function also includes the sense of smell and a loss of this function has a marked effect on both the ability to smell and taste. Nasal breathing is the preferred breathing route at rest for most people, 
but there is a shift to oral breathing at some point when developing nasal obstruction that varies individually. Oral breathing, apart from being less effective than the nose in air conditioning, also promotes airway collapse in oropharynx due to increased upper airway resistance (30).

Assessing nasal patency: Objective measurements of changes in nasal patency can be obtained either as alterations in nasal air flow and resistance or as changes in intranasal diameter and volume. Rhinomanometry, nasal peak flow and nasal spirometry are examples of methods that give a good perception of the overall nasal air flow resistance but are less reliable to predict specific changes in the mucosa. Acoustic rhinometry or rhinostereometry are preferred when studying the variations in the nasal cavity dimensions due to swelling and decongestion at specific sites in the nasal cavity. For instance it has been shown that the minimal cross sectional area at $4 \mathrm{~cm}$ from the nostril is where the nasal mucosa decongests the most (31). Acoustic rhinometry, introduced by Hilberg in the 1980`s is by far the most widely used of the 2 methods and is quick to perform (32). Audible sound is lead into the nasal cavity and reflected back to generate a description of nasal cross sectional areas and volumes at different levels. Attempts have been made to correlate intranasal dimensions in healthy subjects to the subjective sensation of nasal patency, age, gender, BMI and head circumference without success. When used to compare changes in the same individual before and after an intervention, acoustic rhinometry has, however, proven accurate with a high reproducibility (33). In a recent review, Eccles found evidence for the benefit of septoplasty in studies using acoustic rhinometry, before and after surgery (34).

\subsection{Nasal function and sleep}

Nasal obstruction is considered the most important factor that links nasal inflammation to poor sleep but other factors such as the presence of inflammatory mediators affecting the CNS may also contribute. Nasal obstruction due to nasal inflammation is probably multi factorial including altered neurovascular control of the sinusoids, formation of sub mucous edema, secretion of excessive nasal secretions and cicardian changes following the serum cortisol cycle with a peak of nasal congestion in the morning. How these factors interact in nasal obstruction and how it affects sleep still remains unclear. This was recently reviewed by Craig and in summary, most studies showing a relationship between allergic rhinitis and poor sleep have focused on symptoms of nasal congestion rather than objective measurements of nasal patency during sleep (21). In a large north European multicentre study study we also found rhinitis to be an independent risk factor for sleep disturbances in asthma specifically in subjects reporting nasal symptoms every day (35). Studies looking at objective measures of nasal function are rare but Lavie et al found a an increased number of micro arousals during sleep in patients with allergic rhinitis during sleep (36)

When regarding nasal congestion as a cause to poor sleep, one has to consider several options. If the nasal congestion is severe enough it leads to a shift into oral breathing and thus the effect on sleep is due to a change in breathing route. Effects of oral breathing on airway collapse and it's relationship to OSAS goes beyond the focus of this chapter and will not be discussed here. If, however, nasal breathing is maintained during recumbent body position and sleep, it is necessary to consider both an increased airway resistance and changes to the neurovascular control of the nasal mucosa in relation to nasal inflammation. A relationship between nasal obstruction and sleep disordered breathing has been observed mainly on 
subjects with oro pharyngeal narrowing in the awake situation and not in all patients with nasal obstruction, underlining that nasal obstruction is a co-factor affecting sleep in a complex way (37). With regard to the neurovascular regulation of the nasal mucosa, interesting data have emerged recently. Objective measurements of nasal patency during sleep are difficult to obtain without interfering with the sleep pattern. Lebowitz has described a study on 10 subjects doing consecutive acoustic rhinometry measurements during sleep while monitoring sleep stage. The results showed that nasal patency varies with sleep stage exhibiting a marked congestion during REM-sleep and decongestion during non-REM sleep (38). Variable nasal obstruction has been found to play a greater role in the pathophysiology of obstructive sleep apnea syndrome, than conditions associated with a fixed obstruction (39). In healthy subjects the nasal patency is typically reduced when going from sitting to supine body position measured with acoustic rhinometry (40). It has been suggested that this alteration of nasal patency supine is due to an increased hydrostatic pressure in the nasal vasculature supine and thus a passive mechanism (41). Ko measured heart rate variability as a measure of activity in the autonomic nervous system simultaneously with rhinomanometry between sitting and supine in 12 healthy subjects and found a significant correlation between decreased sympathetic activity supine followed by decreased nasal patency indicating an active regulation of nasal patency supine (42). In studies of patients with snoring and OSAS without rhinitis nasal geometry changes less or remain unchanged supine compared to healthy controls $(43,44)$. This could be related to an increased sympathic activity seen in this patient group (45). In fact an increased nasal obstruction or symptoms of nasal obstruction when lying down has been observed in patients with seasonal allergic rhinitis compared to controls, indicating an inflammatory up regulation of the neurovascular control of the nasal mucosa (46 47).

We recently found no response with acoustic rhinometry to a change between sitting and supine in 19 patients with asthma and allergic rhinitis at inclusion, but a normalized reaction (with significantly decreased nasal patency supine) after 6 weeks treatment with a nasal steroid compared to placebo (unpublished data). Disturbances in the neurovascular control of the nasal mucosa supine is thus present in allergic rhinitis along with nasal obstruction and could interfere with nasal function during sleep but this has to evaluated further.

\section{Is there a definite relationship between nasal congestion and SDB?}

The relationship between subjective and objective measures of nasal obstruction are often poor which can confound studies in this area. Nasal obstruction is an independent risk factor for OSAS (9) but this is not confirmed in all studies. In normal subjects occlusion of the nose during the night causes an increase in sleep apnoea and transient arousals (50) suggesting that nasal obstruction irrespective of cause is an important cause of OSAS. In AR there is a general relationship between the presence and degree of nasal obstruction and obstructive sleep apnoea $(8,50,51)$. This has also been confirmed in population studies (17). Anatomical deformities such as nasal septal deviation, adenoidal hypertrophy, nasal polyps are associated with SDB. However there are also other important predisposing factors for OSAS such as obesity, craniofacial abnormalities and male sex. 


\subsection{Does reversal of nasal obstruction also reverse the sleep disorder?}

Physical modalities of reversal of nasal obstruction include surgery to correct nasal septal deformity, removal of nasal polyps and adenoidectomy. The results of septoplasty in a randomised control trial (52) showed that only a subgroup (15\%) benefited in terms of improved OSAS. This is consistent with other studies which often show improvements in some indices eg less sleepiness, better QOL but no changes in the apnoea-hypopnoea index (53). This suggests that OSAS is multifactorial and alleviation of nasal obstruction is only a partial solution. Tonsillectomy and adenoidectomy in children with OSAS can result in complete resolution of symptoms but results are variable between studies with success rates of $27-87 \%$ reported $(54,55)$.

The use of nasal dilators has been examined in 5 studies and has provided equivocal results. Breathe Rite nasal strips: these increase PNIF by a mean of $26 \mathrm{~L} / \mathrm{min}$ and also significantly improve the respiratory index (56)

\subsection{Medical treatment for nasal congestion as treatment for SDB}

Antihistamines (oral or topical) have less effect on nasal congestion than INCS or decongestants but have been shown to be helpful in improving sleep in some studies (57) Oral decongestants do reduce nasal congestion but this is not accompanied by improvements in sleep quality probably due to the side effects of pseudoephedrine (58). Topical decongestants also reduce nasal obstruction and improve sleep but are never recommended for long term use due to the risk of developing rhinitis medicamentosa (59) Leukotriene receptor antagonists are an effective treatment for SAR providing improvements in nasal congestion and also some improvements in sleep impairment (60).

The first line of treatment when treating nasal obstruction in allergic rhinitis with or without concomitant asthma according to the evidence based guidelines Allergic rhinitis and its impact on asthma (ARIA) is intranasal steroids (48). The evidence is 1A for alleviating nasal congestion but nasal steroids also reduce other symptoms of nasal inflammation such as itching, sneezing and secretion along with a reduction of ocular symptoms (49). This is accompanied by improved QOL. In allergic rhinitis patients treated with nasal steroids improved significantly in self reported quality of sleep but not in objective sleep measured with polysomnography and actigraphy $(19,24)$.

\section{Results of current research}

We recently conducted a study utilising objective measures of nasal patency (PNIF, acoustic rhinometry) and sleep parameters (actigraphy) in subjects with mild asthma and PAR. The effect of INCS was also determined.

Nineteen patients with asthma and allergic rhinitis were assessed before and after 6 weeks treatment with an intranasal steroid spray (fluticasone propionate) versus 6 weeks of placebo nasal spray in a double blind cross over design (unpublished data). Nasal patency was measured with acoustic rhinometry sitting and supine and objective quality of sleep was measured with actigraphy. Actigraphy measures limb movements during sleep and has been validated against polysomnography. While actigraphy failed to show any improvement in sleep quality after nasal steroid treatment, there was a significant improvement in rhinitis specific health related quality of life measured with the rhinitis quality of life questionnaire (RQLQ). More importantly we found no change in nasal 
patency between sitting and supine at baseline, indicating an impaired neurovascular control. After 6 weeks treatment with a nasal steroid the nasal reactivity between sitting and supine returned to normal, showing a significant decrease supine compared to sitting. According to ARIA the evidence shows that even though oral antihistamines and leukotriene antagonists have an effect on several symptoms of allergic rhinitis, nasal steroids are the most effective in treating nasal congestion. Our data suggest that nasal steroids also restore neurovascular control in the nasal mucosa, a mechanism that is involved in the adaptation of nasal patency between sitting and supine and in the variation of nasal congestion during sleep. Larger studies are still needed to establish how neurovascular control is affected during wakefulness and during sleep and how nasal obstruction and impaired nasal function interact in patients with allergic rhinitis.

\section{Summary}

Nasal inflammation in allergic rhinitis adversely affects sleep. Nasal congestion is believed to be one of the more important factors based on self reported data and a limited number of studies evaluating nasal function before and after anti inflammatory treatment. Neurovascular dysfunction in the regulation of nasal patency and circulating inflammatory mediators may also contribute to disturbed sleep along with other rhinitis symptoms such as itching and sneezing. Nasal steroids are effective in treating allergic rhinitis and contribute to reducing symptoms of poor sleep. The causes of SDB in Ar are likely to be multifactorial and include a newly named entity RDS. The specific mechanisms behind allergic rhinitis and poor sleep warrant further examination.

\section{References}

[1] Salib RJ, et al Allergic rhinitis: past,present and future. Clin Otolaryngol 2003;28:291-303

[2] Lunn M, Craig T Rhinitis and Sleep. Sleep Medicine Reviews 2011; xxxx:1-7

[3] The Economic Impact of Allergic Disease in Australia: not to be sneezed at. Report by Access Economics for ASCIA 2007. Available on the ASCIA website: www.allergy.org.au

[4] Blaiss $\mathrm{M}$ et al A study to determine the impact of rhinitis on sufferers' sleep and daily routine. JACI 2005;115:S197

[5] Meltzer EO et al Burden of allergic rhinitis: results from the Paediatric Allergies in America survey. JACI 2009;124:S124

[6] Sutherland ER Nocturnal Asthma Journal of Allergy and Clinical Immunology; 2005:116:1179-1186

[7] Stull DE et al Relationship of nasal congestion with sleep, mood and productivity. Curr Med Res Opinion 2007;23:811-819

[8] Santos CB et al Allergic rhinitis and its effect on sleep, fatigue, and daytime somnolence Ann All Asthma Immunol 2006;97:579-586

[9] Staevska MT et al Rhinitis and sleep apnoea. 2004 Curr All Asthma Rep 4:193-199

[10] Meltzer EO 2 et al Sleep, quality of life and productivity impact of nasal symptoms in the United States: findings from the Burden of Rhinitis in America Survey. Allergy Asthma Proc 2009;30: 244-254 
[11] Gupta N et al Allergic rhinitis and Inner-City Children - is there a relationship to sleep disordered breathing? JACI 2007 abstract 606.

[12] Jauregui I et al Allergic rhinitis and school performance J Invest Allergology \& Clin Immunol 2009;19S:32-39

[13] Engel-Yeger B et al Differences in leisure activities between children with allergic rhinitis and healthy peers. Int J of Ped Otorhinolaryngology 2010;74:1415-1418

[14] Sogut A et al Prevalence of habitual snoring and symptoms of sleep disordered breathing in adolescents. Int J of Ped Otorhinolaryngology 2009;73:1769-1773.

[15] Storms W et al The Nasal Allergy Survey Assessing limitations (NASL) 2010 survey: Allergic rhinitis associated with substantial sleep disturbances. JACI 2011;127:abstract 836.

[16] Leger D et al Allergic rhinitis and its consequences on quality of sleep. An unexplored area Arch Int Med 2006;166:1744-1748.

[17] Young $\mathrm{T}$ et al Chronic nasal congestion at night is a risk factor for snoring in a population-based cohort study. Arch Int Med 2001; 161: 1514-1519.

[18] Bender BG and Leung DYM Sleep disorders in patients with asthma, atopic dermatitis and allergic rhinitis. JACI 2005; 116:1200-1201

[19] Rimmer J et al Sleep disturbance in persistent allergic rhinitis measured using actigraphy. Ann Allergy Asthma and Immunol. 2009;103:190-194

[20] Yuksel H et al Sleep actigraphy evidence of improved sleep after treatment of allergic rhinitis.Ann Allergy Asthma and Immunol 2009; 103: 290-294

[21] Craig T, Sherkat A, Safaee S. Congestion and sleep impairment in allergic rhinitis. 2010 Curr Allergy Asthma Rep 10:113-121

[22] Bousquet J et al World Health Organisation: GA(2)LEN;Allergen. Allergic rhinitis and its impact on asthma. (ARIA) 2008 update. Allergy 2008; 63(suppl 86) 8-160.

[23] Smolensky MH et al Sleep disorders, medical conditions, and road accident risk 2011 Accident Analysis and Prevention 43: 533-548

[24] Meltzer EO et at Intranasal mometasone furoate therapy for allergic rhinitis symptoms and rhinitis-disturbed sleep. Ann All Asthma Immunol 2010;105:65-74

[25] National Sleep Foundation: Sleep In America Poll 2008: www.sleepfoundation.org

[26] Stuck BA et al Changes in daytime sleepiness, quality of life, and objective sleep patterns in seasonal allergic rhinitis: A controlled clinical trial. J or Allergy and Clin Immunol:2004: 113:663-8.

[27] Baraniuk J Neural Regulation of Mucosal Function. 2008 Pulm Pharmacol Ther 21(3): 442-448.

[28] Davies AM, Eccles R Reciprocal changes in nasal resistance to air flow caused by pressure applied to the axilla. 1985 Acta Otolaryngol 99:154-159

[29] Svensson S, Hellgren J. PH in Nasal Exhaled Breath Condensate in healthy adults. 2007 Rhinology 45:214-217.

[30] Fitzpatric M, McLean H, Urton A, Tan A, O’Donnell D, Driver H. Effect of nasal and oral breathing route on upper airway resistance during sleep. 2003 Eur Respir J 22:827-832

[31] Grymer LF, Hilberg O, Acoustic rhinometry; values from adults with subjective normal nasal patency. 1991 Rhinology 29(1):35-47.

[32] Hilberg O.Objective measurement of nasal airway dimensions using acoustic rhinometry:methodological and clinical aspects. 2002 Allergy 57 Suppl 70:5-39 
[33] Clement PA, Gordts F Standardisation Committee on Objective Assessment of the Nasal Airway, IRS, and ERS. Consensus report on acoustic rhinometry and rhinomanometry. 2005 Rhinology Sep 43(3):169-179

[34] Moore M, Eccles R. Objective evidence for the efficacy of surgical management of the deviated septum as treatment for chronic nasal obstruction: a systematic review. 2011 Clin Otolaryngol 36:106-113

[35] Hellgren J, Omenaas E, Gíslason T, Jögi R, Franklin K, Lindberg E, Janson C, Torén K. Perennial non-infectious rhinitis-an independent risk factor for sleep disturbances in asthma. 2007 Respir Med. May;101(5):1015-20. Epub 2006 Oct 16.

[36] Lavie P, Gertner R, Zomer J, Podoshin L. Breathing disorders in sleep associated with microarousals in patients with allergic rhinitis. 1981 Acta Otolaryngol 92:529-33.

[37] Pevernagie D, De Meyer M, Claeys S. Sleep breathing and the nose. 2005 Sleep Medicine Reviews 9:437-451

[38] Luc G.T. Morris, MD, Omar Burschtin, MD, Jennifer Setlur, MD,Claire C. Bommelje, MD, Kelvin C. Lee, MD, Joseph B. Jacobs, MD, and Richard A. Lebowitz, MD REMassociated nasal obstruction: A study with acoustic rhinometry during sleep 2008 Otolaryngology-Head and Neck Surgery 139, 619-623

[39] McNicholas WT The nose and OSA: variable nasal obstruction may be more important in pathophysiology than fixed obstruction. 2008 Eur Respir J 32:3-8

[40] Devyani Lal, M.D., Melissa L. Gorges, B.S., Girapong Ungkhara, M.D., Patrick M. Reidy, M.D.,and Jacquelynne P. Corey, M.D. Physiological change in nasal patency in response to changes in posture, temperature, and humidity measured by acoustic rhinometry. 2006 Am J Rhinol 20, 456-462.

[41] Rundcrantz H Postural variations of nasal patency. 1969 Acta Otolaryngol 68:435-443

[42] Ko JH, Kuo TB, Lee GS Effect of postural change on nasal airway and autonomic nervous system established by rhinomanometry and heart rate variability analysis. 2008 Am J Rhinol 22:159-165

[43] Virkkula P, Maasilta P, Hytönen M, Salmi T, Malmberg H Nasal obstruction and sleepdisordered breathing: the effect of supine body position on nasal measurements in snorers. 2003 ActaOtolaryngol 123:648-654

[44] Hellgren J, Yee BJ, Dungan G, Grunstein RR. Altered positional regulation of nasal patency in patients with obstructive sleep apnoea syndrome. 2009 Eur Arch Otorhinolaryngol. Jan;266(1):83-7. Epub 2008 May 14

[45] Phillips CL, Yang Q, Williams A, Roth M, Yee BJ, Hedner JA, Berend N, Grunstein RR The effect of short-term withdrawal from continuous positive airway pressure therapy on sympathic activity and markers of vascular inflammation in subjects with obstructive sleep apnoea. 2007 J Sleep Res 16:217-225

[46] Hasegawa M, Saito Y Postural variations in nasal resistance and symptomatology in allergic rhinitis. 1979 Acta Otolaryngol. 88(3-4):268-72.

[47] Roithmann R, Demenghi P, Faggiano R, Cury A Effects of posture change on nasal patency. 2005 Rev Bras Otorrinolaringol 4:478-484

[48] Brozek JL, Bousquet J, Baena-Cagnani CE, Bonini S, Canonica GW, Casale TB, van Wijk RG, Ohta K, Zuberbier T, Schünemann HJ Allergy and Asthma European Network; Grading of Recommendations Assessment, Development and Evaluation Working Group. Allergic Rhinitis and its Impact on Asthma (ARIA) guidelines: 2010 revision. J Allergy Clin Immunol Sep;126(3):466-760 
[49] Craig T, Hanks CD, Fisher L. How do topical nasal corticosteroids improve sleep and daytime somnolence in allergic rhinitis? 2005 J Allergy Clin Immunol 116:1264-1266

[50] Canova CR et al Increased prevalence of perennial allergic rhinitis in patients with obstructive sleep apnoea. Respiration 2004; 71: 138-141.

[51] Nathan RA et al Objective monitoring of nasal patency and nasal physiology in rhinitis. JACI 2005; 115: S442-59

[52] Koutsourelakis I et al Randomised trial of nasal surgery for fixed nasal obstruction in OSA. Eur Resp J 2008;32:110-117.

[53] Roscow DE et al Is nasal surgery an effective treatment for obstructive sleep apnoea? The Laryngoscope 2010;120:1496-1497.

[54] Ye J et al Outcome of adenotonsillectomy for OSAS in children. Ann Otol Rhinol \& Laryngol 2010;119:506-513.

[55] Bhattacharjee R et al Adenotonsillectomy outcomes in the treatment of OSA in children: a multicentre retrospective study. Am J Resp and Crit Care Med 2010;182:676-683.

[56] Rupp MR et al Breathe Rite Strips improve Respiratory index in patients with rhinitis and OSA. JACI 2006; abstract 291

[57] Murray JJ et al Comprehensive evaluation of cetirizine in the management of seasonal allergic rhinitis: impact on symptoms, quality of life, productivity and activity impairment. All Asthma Proc 2002;23:391-398.

[58] Sherkat AA et al The role of pseudoephedrine on daytime sleepiness in patients suffering from perennial allergic rhinitis. Ann Allergy Asthma Immunol 2011;106:97-102

[59] McLean HA et al Effect of treating severe nasal obstruction on the severity of obstructive sleep apnoea. Eur Resp J 2005; 25: 521-527.

[60] Rodrigo GJ, Yanez A. The role of antileukotriene theray in seasonal allergic rhinitis: A systemic review of randomised trials. Ann Allergy Asthma Immunol. 2006;96:779786. 


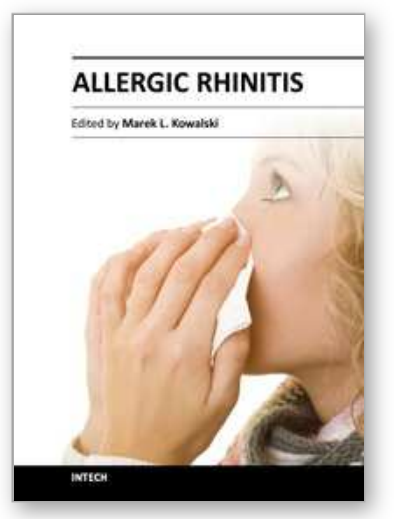

\author{
Allergic Rhinitis \\ Edited by Prof. Marek Kowalski
}

ISBN 978-953-51-0288-5

Hard cover, 214 pages

Publisher InTech

Published online 21, March, 2012

Published in print edition March, 2012

Allergic rhinitis, while troublesome for a patient, may be also a challenge for the physician. That is why physicians must still learn more on the pathophysiology, clinical spectrum and novel diagnostic and therapeutic approaches to the disease. The chapters of this volume address a variety of important topics related to allergic rhinitis. They begin with a description of innovative translational approaches allowing for unification of animal and human models. Contributing authors provide up-to-date reviews of clinical aspects of allergic rhinitis in children, its association with bronchial asthma and other co-morbid conditions. They also discuss the impact of allergic rhinitis on sleep and sports. Together with articles on diagnostic approaches as well as novel treatments, the book offers a comprehensive and stimulating review of the topic. May this book find a wide readership among allergists and other physicians interested in allergic disease, and also among pediatricians, general practitioners and other specialists who increasingly have to deal with this seemingly benign, but sometimes extremely troublesome, disease.

\title{
How to reference
}

In order to correctly reference this scholarly work, feel free to copy and paste the following:

J. Rimmer and J. Hellgren (2012). Allergic Rhinitis and Its Impact on Sleep, Allergic Rhinitis, Prof. Marek Kowalski (Ed.), ISBN: 978-953-51-0288-5, InTech, Available from: http://www.intechopen.com/books/allergicrhinitis/allergic-rhinitis-and-its-impact-on-sleep

\section{INTECH}

open science | open minds

\section{InTech Europe}

University Campus STeP Ri

Slavka Krautzeka 83/A

51000 Rijeka, Croatia

Phone: +385 (51) 770447

Fax: +385 (51) 686166

www.intechopen.com

\section{InTech China}

Unit 405, Office Block, Hotel Equatorial Shanghai

No.65, Yan An Road (West), Shanghai, 200040, China 中国上海市延安西路65号上海国际贵都大饭店办公楼405单元

Phone: +86-21-62489820

Fax: $+86-21-62489821$ 
(C) 2012 The Author(s). Licensee IntechOpen. This is an open access article distributed under the terms of the Creative Commons Attribution 3.0 License, which permits unrestricted use, distribution, and reproduction in any medium, provided the original work is properly cited. 